\title{
Framework for modeling and simulation of household appliances
}

\author{
Christian Bjerknes Nilsen \\ Department of Electrical Engineering \\ UiT - The Artic University of Norway \\ Narvik, Norway \\ christian.bjerknes7@hotmail.com
}

\author{
Bjarte Hoff \\ Department of Electrical Engineering \\ UiT - The Artic University of Norway \\ Narvik, Norway \\ bjarte.hoff@uit.no
}

\author{
Trond Østrem \\ Department of Electrical Engineering \\ UiT - The Artic University of Norway \\ Narvik, Norway \\ trond.ostrem@uit.no
}

\begin{abstract}
This paper describes a framework for simulating household appliances in Simulink. Typical Norwegian household equipment have been analyzed based on their characteristic behavior, to establish the requirement for synthesizing and recreating their current waveform, harmonic currents and phase shift. Both detailed simulation of the current waveform and RMS currents for simulation over longer time periods are implemented. Models for several appliances can be integrated with the Simscape Power System toolbox in Simulink and connected to single-phase or threephase grid models. Simulations are verified with experimental measurements for a selection of household appliances.
\end{abstract}

Keywords—Load modeling, home appliances, Fourier series, simulation.

\section{INTRODUCTION}

The electric power grid has existed for a long time with minimal changes. The grid-connected equipment has not remained unchanged. Especially the use of power electronic devices is increasing rapidly. Nowadays, power electronics is present in everything from LED lights, computers and refrigerators to electric cars and renewable energy production. With every new device, the relationship between power, current and voltage may differ. Disturbances and harmonic currents can also introduce challenges with respect to power quality. Household appliances accounts for a significant part of the load in residential areas. It is important to understand how new types of appliances will affect the future grid, especially in remote areas with weak grid connection or in microgrids.

The increasing use of efficient and high-power devices, such as induction cookers, has stimulated to research on demand response and scheduling algorithms in order to reduce power peaks from households [1-3]. However, lowpower devices with a high content of harmonic currents also affect power quality. Devices such as certain LED lamps will exhibit a constant power characteristic, where a reduction in load voltage results in an increased load current. Too high penetration of constant power loads can pose a challenge in grid stability [4]. Consequently, modelling of household appliances is important in order to investigate how the load characteristics may change in the future.

Statistical methods have previously been applied for analyzing active power consumption for entire households in [5], whereas harmonics in residential networks are modelled in [6]. The possibility to classify household appliances by

The paper is a part of the Arctic Energy project funded by the Interreg Nord EU-program, and Nordland and Troms County Council, Norway analyzing the active power waveforms has been demonstrated in [7], based on load profiles from 27 households recorded over one year.

Simulation of individual household appliances is shown in $[8,9]$, but the simulations are based on a detailed modelling approach on component level. Due to the small time-step required to reproduce nonlinear behavior, it would be challenging to extend the method to an entire household over longer simulation periods like days or weeks.

This paper presents a method of modelling and simulating multiple appliances in a household using Simulink. The simulation method synthesizes the load current waveform of a household, with the possibility to integrate it in a larger power system analysis or simulation.

The paper is organized as follows: section II explains the mathematical model, section III and IV discuss common load types and their simulation requirements, while the Simulink implementation is shown in section V. Experimental verification is presented in section VI, and section VII concludes the paper.

\section{MATHEMATICAL MODEL}

Modelling of household appliances must be able to regenerate the current waveform, displaying active and reactive power, as well as deviations from the sine wave. Linear models in the form of transfer functions and statespace systems will not be able to capture nonlinear elements in the appliances. Detailed knowledge of the appliances construction will not be available in most cases, as manufactures generally do not supply electrical circuit diagrams. Hence, the detailed modelling using fundamental components (e.g. diodes, transistors) as in [8, 9], is not suitable for a general household appliance simulation model.

Accordingly, this paper will base the model on a Fourier series, where the appliance load current can be synthesized based on amplitude and phase for each harmonic component. The Fourier series in the model can be described as follows:

$$
i_{\text {synth }}(t)=I_{0}+\sqrt{2} \sum_{k=1}^{\infty}\left[I_{k} \sin \left(k \omega_{1} t+\phi_{k}\right)\right] \text {, }
$$

where $\omega_{1}=2 \pi f$ is the fundamental frequency and $k$ is an integer equal to or larger than one.

The coefficients $I_{d c}, I_{k}$ and $\phi_{k}$ are acquired for each appliance through performing a fast Fourier transform (FFT) 
on the measured load currents, where $I_{k}$ represents the RMS current and $\phi_{k}$ is the phase shift for each harmonic component in the model. $I_{0}$ is the $0 \mathrm{~Hz}$ component and introduces an offset in the current waveform. In a practical AC system, this component will gradually decay.

Instead of synthesizing the current waveform, RMS values can be calculated based on the above-mentioned coefficients using:

$$
I_{R M S}(t)=\left(I_{0}^{2}+\sum_{k=1}^{\infty} I_{k}^{2}\right)^{\frac{1}{2}} .
$$

\section{ANALYSIS OF LOADS AND THEIR REQUIREMENTS}

There are many different household appliances, each with their own distinctive current waveform. The current for each appliance is analyzed based on its harmonic content. By comparing the measured current with the synthesized one, the required number of harmonic components (highest number of $k$ ) can be identified.

For the analysis, the appliances are sorted in the following main categories:

- $\quad$ Single phase rectifier load

- Impedance load

- Other loads

\section{A. Single phase rectifier load}

One of the more demanding loads in the households is the rectifier load, found in the majority of small power supplies. Mobile phone chargers, TVs and certain LED lamps are examples of equipment that falls into this category.

The current waveform is characterized by sharp peaks due to charging of the DC capacitor inside the equipment. When the grid voltage is higher than the DC capacitor voltage (close to the maximum positive of negative voltage value), the rectifying diodes start conducting and give the characteristic charging current. The dashed line in Fig. 1 shows a simulated diode rectifier using the SimPowerSystem toolbox in Simulink. An FFT analysis is thereby performed on the simulated current, identifying its harmonic coefficients. The synthesized current using the Fourier series in (1) is shown as solid lines in Fig. 1 for different number of harmonic components.

In Fig. 2, the error between the simulated and synthesized current waveform is shown for an increasing number of harmonics components. The cumulative error over one period $\mathrm{T}$ indicates how the result will deviate over time, as it includes the sign of the error:

$$
e_{\text {cum }}=\sum_{t=0}^{T}\left[i_{\text {sim }}(t)-i_{\text {synth }}(t)\right] .
$$

The quadratic error represents the difference in shape:

$$
e_{\text {quad }}=\sum_{t=0}^{T}\left[i_{\text {sim }}(t)-i_{\text {synth }}(t)\right]^{2} .
$$

The synthesized current will approach the original simulation result as the number of harmonic components is increased.
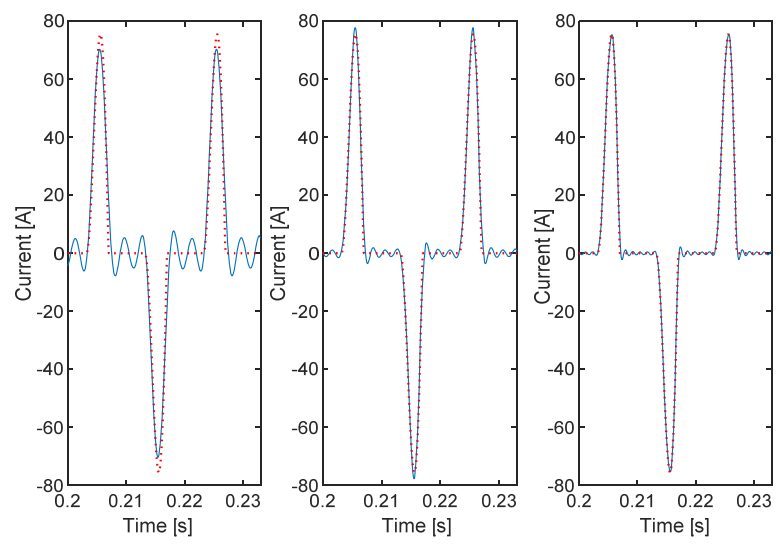

Fig. 1. Synthesized rectifier current with up to a) $5^{\text {th }}$ harmonic, b) $10^{\text {th }}$ harmonic c) $15^{\text {th }}$ harmonic. Simulated waveform is dashed.

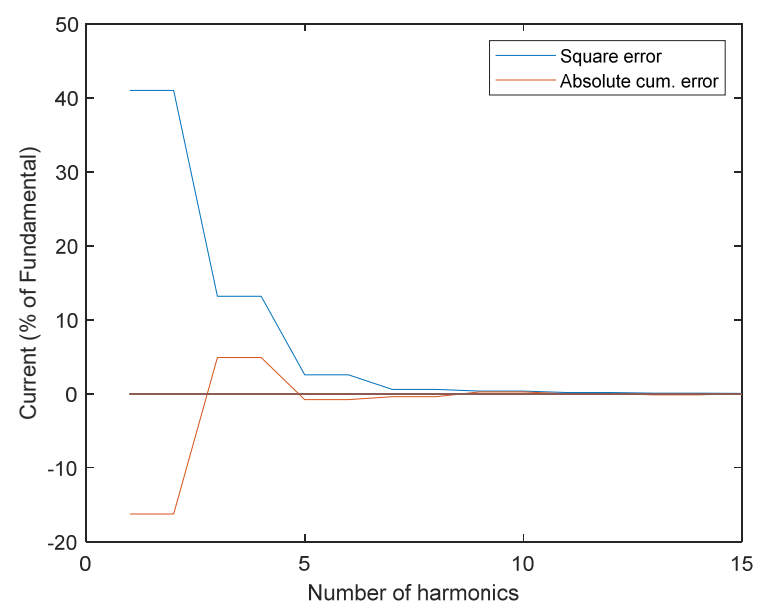

Fig. 2. Quadratic and cumulatove error as functions of harmonic spectrum, where $50 \mathrm{~Hz}$ is defined as findamental frequency.

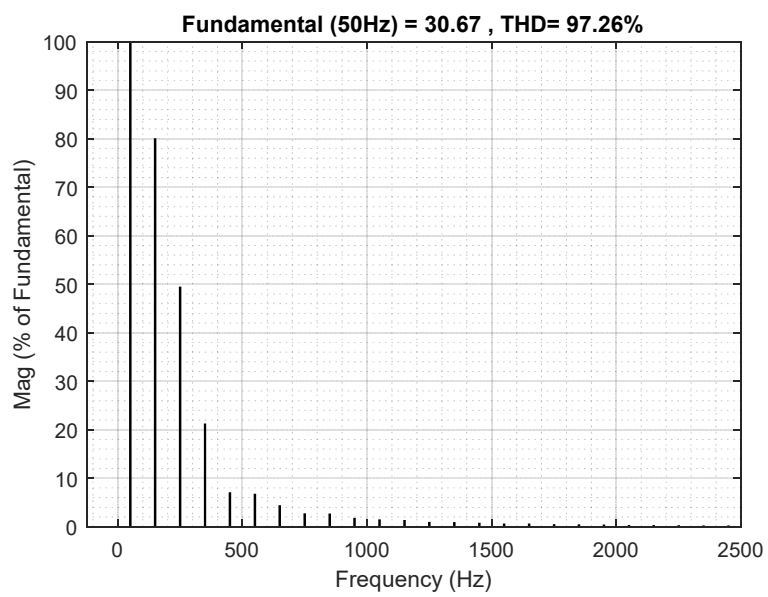

Fig. 3. The frequency spectrum for a simulated rectifier.

As shown in Fig. 2 and 3, the spectrum up to $750 \mathrm{~Hz}$ contains most of the frequency components. By including up to the 15 th harmonic in the synthesis, a cumulative error of $0.039 \%$ and quadratic error of $0.085 \%$ is achieved.

For rectifier loads, it is therefore considered sufficient to include up to the $15^{\text {th }}$ harmonic $(750 \mathrm{~Hz})$ in the model. 


\section{B. Impedance load}

An impedance load is considered linear, with a pure sine current. Typical appliances are hot water tanks and electric heating devices. The current can be phase shifted if containing capacitive or inductive elements. Hence, only the fundamental frequency component $I_{1}$ is required in order to synthesize the current waveform:

$$
\mathrm{i}_{\text {synth,imp }}(\mathrm{t})=\sqrt{2} \mathrm{I}_{1} \sin \left(\mathrm{k \omega 1} t+\phi_{1}\right)
$$

\section{Other loads}

In addition to rectifier and impedance loads, households include loads like single-phase motors, microwave ovens, light fixtures, refrigerators etc. These are categorized as other non-linear loads. In a single-phase motor, the core saturation will generate current harmonics with a pronounced third harmonic component. The magnetron in the microwave oven is also a source of current harmonics, as is the discharge lamps, due to the nonlinear characteristics of the interior discharge. In some cases, electric motors are controlled by power electronics, e.g. universal motors with triac control, and in such cases the electronics would add to the total current harmonics.

An example of current waveforms for a selection of typical appliances are shown in Fig. 4, where the rectifier load found is found in the CDC-5050 receiver, $5 \mathrm{~W}$ LED and the Samsung phone charger (EP-TA20EW). Since the rectifier described in section II.A is considered as the most demanding case in terms of distorted current, a spectrum up to the $15^{\text {th }}$ harmonic will be set as the requirement for other load types as well.

\section{GRID VOLTAGE FLUCTUATIONS}

Due to impedance in cables and wires, small voltage dips and swells are expected during normal grid operation. In addition comes larger voltage deviations due to faults.

Load behavior as functions of load voltage changes is normally modelled using the ZIP model, describing the change in active and reactive power $[4,10]$. The model is considered valid up to $30 \%$ deviation from nominal voltage. In (6), the load current characteristics has been derived from the original ZIP model:

$$
i(t)=i_{\text {synth }}(t)[\underbrace{a_{1}\left(\frac{V}{V_{0}}\right)}_{Z_{\text {const }}}+\underbrace{a_{2}}_{I_{\text {const }}}+\underbrace{a_{3}\left(\frac{V_{0}}{V}\right)}_{P_{\text {const }}}],
$$

where the coefficients $a_{1}, a_{2}$ and $a_{3}$ determines if the load characteristic is constant impedance, current or power, $i_{\text {synth }}(t)$ is the synthesized load current from the Fourier series in (1) at nominal voltage $V_{0}$, and $V$ is the RMS load voltage.

These three ZIP model elements are demonstrated in Fig. 5 , where the ZIP model is applied on a purely linear load. Grid voltage drops from $230 \mathrm{~V}$ to $207 \mathrm{~V}$ at $\mathrm{t}=0.5$. As expected, an impedance load component will respond with a reduction in load current due to the voltage drop, while the constant power load component will increase the current.

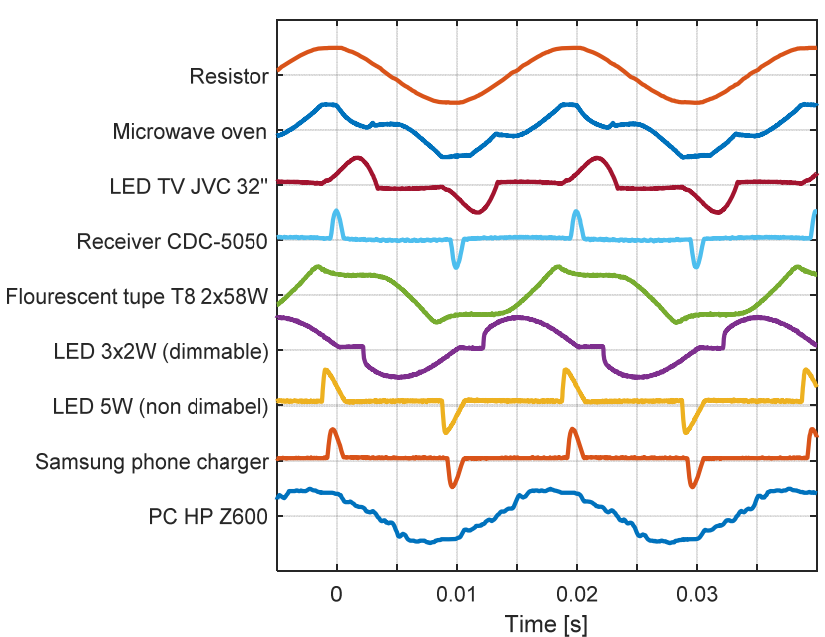

Fig. 4. Measured current waveforms for a selection of typical household appliances.
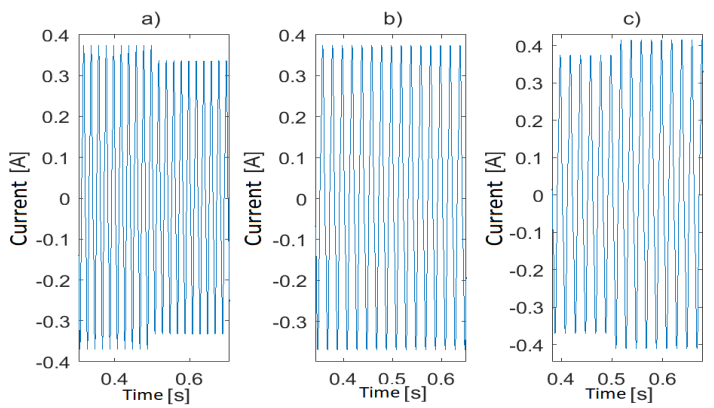

Fig. 5. Load current with varying grid voltage for, a) constant impedance, b) constant current and c) constant power.

In addition to voltage drops from impedance in cables, distorted current waveforms (i.e. harmonic currents) can affect the grid voltage, since circulating currents may appear. This will vary depending on the connected appliances and grid configuration. Such phenomena can be identified by analyzing simulation results.

\section{SIMULLINK IMPLEMENTATION}

The model described in section IV and V is implemented in Simulink in order to create a framework for simulating households. Fig. 6 shows the model layout, where grid RMS voltage is used as input in order to allow output current changes according to (6). An additional control input is used in order to allow external controllers to turn on or off or to scale the load current component:

$$
i_{\text {out }}(t)=i(t) \cdot c
$$

where $c$ is the control input.

The control input also allows modelling of appliances that contain several load types, such as the dishwasher model shown in Fig 7. By combining several sub-models and activate them in a sequence, even time varying appliances can be modelled.

While this response is obvious from (6), it is of great interest to investigate the characteristics when multiple models are combined into an entire household model. Knowledge of future household load characteristics can shed light on future voltage stability challenges in the grid. 


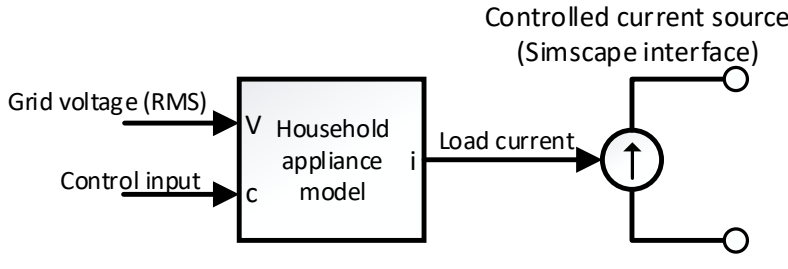

Fig. 6. Simulink implementation of the household applience model.

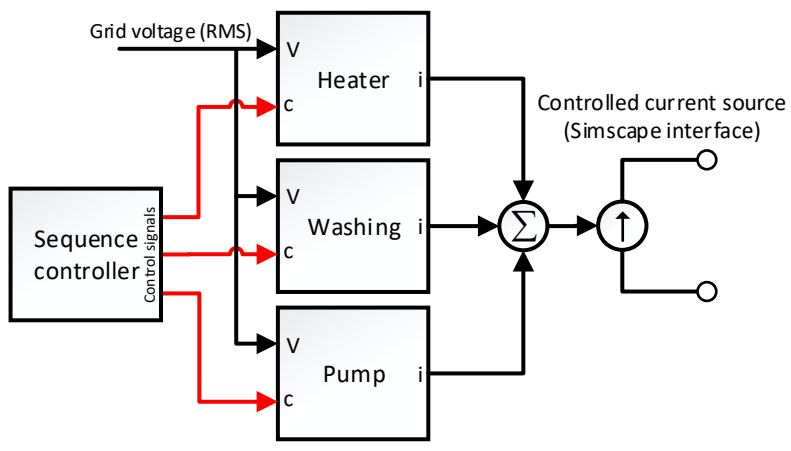

Fig. 7. Example of dishwasher model.

For convenience, the synthesized current can be used to control a "current source" in the Simulink Simscape environment, and appliance models can be connected to both single-phase and three-phase model grids. Note that a phase shift should be added for three-phase grid simulations, depending on which phases the model is connected to.

The implemented model is set to acquire the amplitude and phase from a predefined matrix in MATLAB Workspace. Harmonic components up to the $20^{\text {th }}$ harmonic are defined in the matrix, which is equivalent to $1000 \mathrm{~Hz}$ in a $50 \mathrm{~Hz}$ grid. Hence, the simulation time step in Simulink should be $100 \mu$ s or less in order to properly synthesize the current waveform.

If the RMS approach in (2) is used, time step can be chosen freely by the user, depending on the application of the simulation model.

\section{EXPERIMENTAL VERIFICATION}

The simulation model is verified by first performing current measurements and FFT analysis on three selected appliances.

The load current of each appliance is recorded using an Agilent InfiniiVision DSO-2002A oscilloscope with the experimental setup shown in Fig. 8. Current is measured using a PROSyS CP30 clamp, while grid voltage is measured using LeCroy AP030 differential probe at 1:100 ratio. Each recording is collecting 2000 samples with a sample time of $100 \mu \mathrm{s}$ and a measurement duration of $200 \mathrm{~ms}$. FFT is performed for the entire recording using a rectangular window. Based on these recordings, amplitudes and phaseshifts up to the $20^{\text {th }}$ harmonic are used in the Simulink model. The grid voltage is $230 \mathrm{~V}$ AC, where the voltage measurement is used as a reference for identifying the correct phase-shift of the load current.

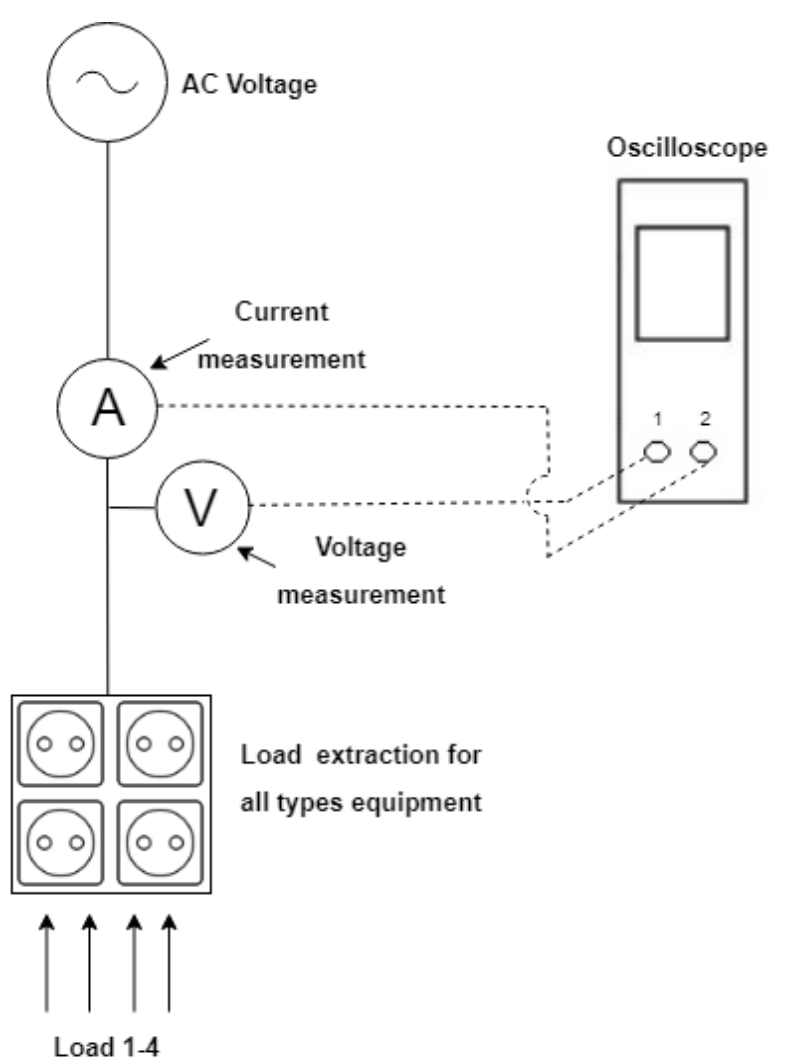

Fig. 8. Experimental setup.

\section{A. Verification of one appliance}

In this case, the load current of a $1.15 \mathrm{~kW}$ microwave oven is measured and compared to the syntesized current from the simulation model.

As shown in Fig. 9, the synthesized current from the model is a close match to the measured current, despite the distorted and nonlinear waveform. The largest difference between simulated and measured current is $288 \mathrm{~mA}$ at $\mathrm{t}=$ $0.1288 \mathrm{~s}$, which equals to $4.85 \%$ of the RMS current value.

\section{B. Verification of multiple appliances}

When simulating households, it is necessary to combine several appliances in order to simulate the total household current. In this experiment, two appliances are measured and analyzed separately, using FFT as described in the beginning of section VI.

A SPS-606 $500 \mathrm{~W}$ (900 VA) laboratory power supply and a $36 \mathrm{~W}$ desktop fan are used for this test, since they have similar current amplitude, avoiding that one appliance dominates the result. Note that the power supply is running at idle with do external load connected. The load currents from the two models are synthesized and added together. A new measurement is performed, with both appliances operating in parallel. As shown in Fig. 10, the simulation result is a close match with the measurement. The maximum difference between simulated and measured values is $20 \mathrm{~mA}$ at $\mathrm{t}=$ $0.1461 \mathrm{~s}$, which is $7.63 \%$ of the RMS current value. 


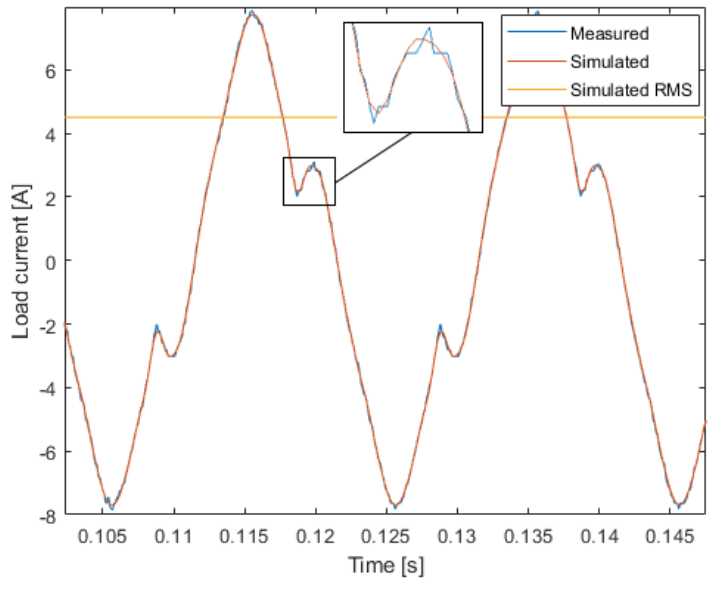

Fig. 9. Microwave oven $(1,15 \mathrm{~kW})$ load current, measured and simulated.

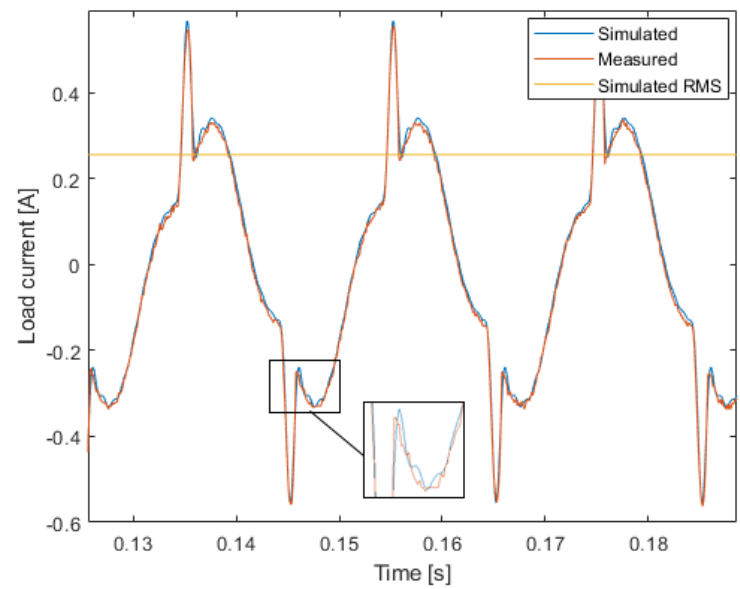

Fig. 10. Measured and simulated laboratory power supply and desktop fan.

\section{CONCLUSION}

A mathematical model and method for simulating household appliances have been demonstrated in this paper.

By creating a library of appliances based on FFT analysis of their load current, the current waveform can be synthesized for each device, without the need of detailed modelling, as well as knowledge of the internal construction.
Several models can thereby be combined in order to simulate the current waveform of an entire household. This allows for active and reactive power analysis, in addition to current harmonics and distortion. By implementing an additional ZIP-model, the household's response to small voltage deviations can be analyzed.

The simulations have been successfully verified through experimental setups for a selection of appliances.

\section{REFERENCES}

[1] M. Pipattanasomporn, M. Kuzlu, and S. Rahman, "An Algorithm for Intelligent Home Energy Management and Demand Response Analysis," IEEE Transactions on Smart Grid, vol. 3, no. 4, pp. 2166-2173, 2012.

F. A. Qayyum, M. Naeem, A. S. Khwaja, A. Anpalagan, L. Guan, and B. Venkatesh, "Appliance Scheduling Optimization in Smart Home Networks," IEEE Access, vol. 3, pp. 2176-2190, 2015.

[3] S. L. Arun, A. A. Raj, and M. P. Selvan, "Demand response in smart buildings through time-varying priority of household appliances," in 2017 IEEE Region 10 Symposium (TENSYMP), 2017, pp. 1-5.

[4] J. Machowski, J. W. Bialek, J. R. Bumby, and J. Machowski, Power system dynamics : stability and control, 2nd ed. ed. Chichester: Wiley, 2008.

[5] C. Wagner, C. Waniek, and U. Häger, "Modeling of household electricity load profiles for distribution grid planning and operation," in 2016 IEEE International Conference on Power System Technology (POWERCON), 2016, pp. 1-6.

[6] G. Ye, M. Nijhuis, V. Cuk, and J. F. G. Cobben, "Appliancebased residential harmonic load modelling," in 2017 IEEE Manchester PowerTech, 2017, pp. 1-6.

[7] D. Li, T. F. Bissyandé, S. Kubler, J. Klein, and Y. L. Traon, "Profiling household appliance electricity usage with $\mathrm{N}$-gram language modeling," in 2016 IEEE International Conference on Industrial Technology (ICIT), 2016, pp. 604-609.

[8] M. J. H. Rawa, D. W. P. Thomas, and M. Sumner, "Experimental Measurements and Computer Simulations of Home Appliances Loads for Harmonic Studies," in 2014 UKSim-AMSS 16th International Conference on Computer Modelling and Simulation, 2014, pp. 340-344.

[9] J. Molina and L. Sainz, "Model of Electronic Ballast Compact Fluorescent Lamps," IEEE Transactions on Power Delivery, vol. 29, no. 3, pp. 1363-1371, 2014.

[10] C. S. Indulkar and K. Ramalingam, "Load Flow Analysis with Voltage-Sensitive Loads," in 2008 Joint International Conference on Power System Technology and IEEE Power India Conference, 2008, pp. 1-6. 